\title{
Chiral extrapolation of the strangeness changing scalar $K \pi$ form factor
}

\author{
Véronique Bernard ${ }^{a}$ and Emilie Passemar ${ }^{b}$ \\ ${ }^{a}$ Groupe de Physique Théorique, IPN, Université de Paris Sud-XI/CNRS, \\ F-91406 Orsay, France \\ ${ }^{b}$ Departament de Física Teòrica, IFIC, Universitat de València - CSIC, \\ Apartat de Correus 22085, E-46071 València, Spain \\ E-mail: bernard@ipno.in2p3.fr, emilie.passemar@ific.uv.es
}

ABSTRACT: We perform a chiral extrapolation of lattice data on the scalar $K \pi$ form factor and the ratio of the kaon and pion decay constants within Chiral Perturbation Theory to two loops. We determine the value of the scalar form factor at zero momentum transfer, at the Callan-Treiman point and at its soft kaon analog as well as its slope. Results are in good agreement with their determination from experiment using the standard couplings of quarks to the $\mathrm{W}$ boson. The slope is however rather large. A study of the convergence of the chiral expansion is also performed.

KEYWORDS: Lattice QCD, Chiral Lagrangians

ARXIV EPRINT: 0912.3792 


\section{Contents}

$\begin{array}{llr}1 & \text { Introduction } & 1\end{array}$

2 ChPT to two loops $\quad 2$

$\begin{array}{llc}3 & \text { Lattice } & 6\end{array}$

$\begin{array}{lll}4 & \text { Chiral extrapolation } & 7\end{array}$

5 Conclusion $\quad 13$

\section{Introduction}

In recent years lots of progress has been made in QCD lattice calculations. One important progress in the light quark sector concerns the values of the quark masses that can now be reached. These are very close to the physical ones making a controlled, i.e. trustable, chiral extrapolation of the lattice results to the physical points possible. A very powerful model-independent framework to perform this extrapolation is Chiral Perturbation Theory (ChPT), the Effective Field Theory of QCD at low energies. Indeed it allows to calculate low-energy QCD processes in terms of the light pseudoscalar mesons masses. Hadron properties are presently actively studied on the lattice and chiral extrapolations to their physical values are performed, see for example [1-3].

At the same time lots of effort is put into testing the Standard Model (SM). In order to do so one has to have very precise determinations of the QCD quantities which generally enter the different processes under consideration. Two very interesting quantities in this respect are the strangeness changing scalar $f_{0}$ and vector $f_{+}$form factors which are measured in $K_{l 3}$ decays [4]. Indeed a measurement of the $K_{\ell 3}$ inclusive decay rate leads to the extraction of the product of the vector form factor at zero momentum transfer $f_{+}(0)$ and of the CKM matrix element $\left|V_{u s}\right|$. Consequently the knowledge of $f_{+}(0)$ allows to extract this matrix element and thus to test the unitarity relation between the elements of the first row of this matrix. Another test comes from the values of this form factor at the Callan-Treiman point [5] and at its soft-kaon analog [6]. Indeed, at these particular points the scalar form factor has a well-known value as dictated by $\mathrm{SU}\left(N_{f}\right) \times \mathrm{SU}\left(N_{f}\right)$ low energy theorems, with $N_{f}=2$ and $N_{f}=3$, respectively. Combining this information with experimental results from semi-leptonic decays one can determine the values of the scalar form factor at these two points in the SM. Thus a departure from these values would be a sign for physics beyond the SM such as right-handed quark couplings to the $W[5,7]$ or charged Higgs effects, see for example the discussion in [4](and references therein) and [8]. However, in order to have a reliable and accurate test of the SM one should know very 
precisely the corrections to the Callan-Treiman theorem and its soft kaon analog which are only exact in the soft meson limit. They are usually calculated in ChPT [9]. In ref. [6] the one-loop result from ref. [9] was used and an estimation of the higher order effects was done since at next-to-leading order some low-energy constants (LECs) contribute which are not very precisely known at present. Experimentally there has recently been interest in trying to obtain the value of the scalar form factor at the Callan-Treiman point. The three collaborations NA48 [10], KLOE [11] and KTeV [12] have reanalysed their data so as to extract this value using in their analysis a dispersive representation of the form factors proposed in refs. $[5,6]$. With the current experimental precision the data from the last two collaborations show a good/marginal agreement with the SM while NA48 has a $4.5 \sigma$ deviation.

The scalar form factor has been studied on the lattice. Some parameterization of its momentum-dependence plus the knowledge of the one-loop ChPT result at zero momentum transfer is used to extract $f_{+}(0)$. Here we will fit the lattice data from ref. [13] for the scalar form factor using a ChPT calculation at two loop order [14]. Furthermore, we will not only consider the scalar form factor but at the same time we will fit the ratio of the kaon to the pion decay constants $F_{K} / F_{\pi}[15,16]$ since, as we will see, similar LECs enter the two quantities. This will allow us to determine some LECs at two-loop order $\left(\mathcal{O}\left(p^{6}\right)\right)$ and thus not only obtain $f_{+}(0)$ and determine $\left|V_{u s}\right|$ but also the value of the scalar form factor at the Callan-Treiman point and at its soft-kaon analog. Of course one should keep in mind that we are dealing here with $\mathrm{SU}(3)$ quantities which involve the strange quark mass. The question is whether one should consider the strange quark as light compared to the QCD scale $\Lambda \sim 200 \mathrm{MeV}$ or should it be treated as heavy. Related to that is the question whether standard $\mathrm{SU}(3)$ ChPT which assumes that the quark condensate is large, is a well converging series, the relevant expansion parameter being in that case $\left(m_{K} / \Lambda_{\chi}\right)^{2} \sim 0.4^{2}$. Also $\bar{s} s$ sea quark pairs may play a significant role in chiral dynamics leading to different patterns of chiral symmetry breaking in $N_{f}=2$ and $N_{f}=3$ chiral limits [17-21]. For example, lattice QCD seems to indicate a problem in the extrapolation of $F_{K} / F_{\pi}$ to its physical value when using SU(3) ChPT to one loop order [16] while a fit within "Kaon ChPT" [22] where the kaon is treated as a heavy particle (for an application of that framework see for example [23]) leads to good agreement. The mass dependence of the scalar form factor has been studied within this scheme in ref. [24]. We will use here standard ChPT to two loops and we will study the convergence of the chiral expansion. We will also discuss the leading order $\mathcal{O}\left(p^{4}\right)$ LEC $L_{4}^{r}$ which is related to the Okubo-Zweig-Iizuka (OZI) rule violation.

In section 2, we discuss briefly the scalar form factor at two loops in ChPT. We present the lattice calculations in section 3 and discuss our fits and results in section 4 . We conclude in section 5 .

\section{ChPT to two loops}

The strangeness changing form factors are defined from the $K \rightarrow \pi$ matrix element of the vector current $V_{\mu}=\bar{s} \gamma_{\mu} u$

$$
\left\langle\pi\left(p_{\pi}\right)\left|\bar{s} \gamma_{\mu} u\right| K\left(p_{K}\right)\right\rangle=\left(p_{\pi}+p_{K}\right)_{\mu} f_{+}(t)+\left(p_{K}-p_{\pi}\right)_{\mu} f_{-}(t),
$$


where $t \equiv q^{2}=\left(p_{K}-p_{\pi}\right)^{2}$. The vector form factor $f_{+}(t)$ represents the P-wave projection of the crossed channel matrix element $\left\langle 0\left|\bar{s} \gamma_{\mu} u\right| K \pi\right\rangle$ whereas the S-wave projection is described by the scalar form factor defined as

$$
f_{0}(t)=f_{+}(t)+\frac{t}{m_{K}^{2}-m_{\pi}^{2}} f_{-}(t) .
$$

At zero momentum one has

$$
f_{0}(0)=f_{+}(0)
$$

These form factors were calculated to two loops in ChPT in ref. [14]. These authors introduced the quantity

$$
\tilde{f}_{0}(t)=f_{+}(t)+\frac{t}{m_{K}^{2}-m_{\pi}^{2}}\left(f_{-}(t)+1-\frac{F_{K}}{F_{\pi}}\right)=f_{0}(t)+\frac{t}{m_{K}^{2}-m_{\pi}^{2}}\left(1-\frac{F_{K}}{F_{\pi}}\right) .
$$

The two-loop expressions of the two decay constants $F_{K}$ and $F_{\pi}$ can be found in ref. [15]. They involve two $L_{i}, L_{4}^{r}$ and $L_{5}^{r}$, at $\mathcal{O}\left(p^{4}\right)$ and the four $\mathcal{O}\left(p^{6}\right) C_{i}, C_{14}^{r}, C_{15}^{r}, C_{16}^{r}$ and $C_{17}^{r}$. Assuming that the LEC $L_{4}^{r}$ is small, which is in principle the case in the standard scenario of ChPT, one can expand, as usually done, the denominator in the ratio of the two decay constants so that its contribution to order $p^{4}$ cancels and one is left with the contribution from $L_{5}^{r}$ and two combinations of three $C_{i}$ as detailed below:

$$
\begin{aligned}
F_{K} / F_{\pi}= & 1+\frac{4}{F_{\pi}^{2}}\left(m_{K}^{2}-m_{\pi}^{2}\right) L_{5}^{r}+\frac{8}{F_{0}^{2}}\left[-m_{\pi}^{4}\left(C_{15}^{r}+2 C_{17}^{r}\right)\right. \\
& \left.+2 m_{\pi}^{2} m_{K}^{2}\left(-\left(C_{14}^{r}+C_{15}^{r}\right)+\frac{1}{2}\left(C_{15}^{r}+2 C_{17}^{r}\right)\right)+2 m_{K}^{4}\left(C_{14}+C_{15}\right)\right]+\delta,
\end{aligned}
$$

where $F_{0}$ is the pion decay constant in the chiral limit. We will come back to the discussion of this equation in section $4 . \delta$ contains the loops and the contributions of the $L_{i}$ at $\mathcal{O}\left(p^{6}\right)$. Interestingly the dependence on these LECs is exactly the same in $f_{0}(t)$, see ref. [14]. Thus the main advantage in considering $\tilde{f}_{0}$ is that this quantity has no dependence on the $L_{i}^{r}$ at order $p^{4}$, only via order $p^{6}$ contributions and furthermore, it only depends on the two $\mathcal{O}\left(p^{6}\right)$ LECs $C_{12}^{r}$ and $C_{34}^{r}$. Its explicit dependence on those is given by

$$
\begin{aligned}
\tilde{f}_{0}(t)= & 1-\frac{8}{F_{0}^{2}}\left(C_{12}^{r}+C_{34}^{r}\right)\left(m_{K}^{2}-m_{\pi}^{2}\right)^{2}+8 \frac{t}{F_{0}^{2}}\left(2 C_{12}^{r}+C_{34}^{r}\right)\left(m_{K}^{2}+m_{\pi}^{2}\right) \\
& -\frac{8}{F_{0}^{2}} t^{2} C_{12}^{r}+\bar{\Delta}(t)+\Delta(0),
\end{aligned}
$$

where we used the notations of ref. [14]. As before, the quantities $\bar{\Delta}(t)$ and $\Delta(0)$ have contributions from loops and from the LECs $L_{i}^{r}$ at $\mathcal{O}\left(p^{6}\right)$ and can in principle be calculated to order $p^{6}$ accuracy with the knowledge of the $L_{i}^{r}$ to order $p^{4}$. Parameterizations of these quantities in the physical region of $K_{\ell 3}$ decays can be found in ref. [14].

Eq. (2.4) is in fact inspired by the Callan-Treiman theorem $[25,26]$ which predicts the value of $f_{0}(t)$ at the so called Callan-Treiman point, $t \equiv \Delta_{K \pi}=m_{K}^{2}-m_{\pi}^{2}$ in the $\mathrm{SU}(2) \times \mathrm{SU}(2)$ chiral limit. One has

$$
f_{0}\left(\Delta_{K \pi}\right)=\frac{F_{K}}{F_{\pi}}+\Delta_{C T},
$$


where $\Delta_{C T}$ is a correction of $\mathcal{O}\left(m_{u, d}\right)$. It has been estimated within ChPT at next-toleading order (NLO) in the isospin limit [9] with the result

$$
\Delta_{C T}^{N L O}=(-3.5 \pm 8.0) \cdot 10^{-3},
$$

where the error is a conservative estimate assuming some typical corrections of $\mathcal{O}\left(m_{u, d}\right)$ and $\mathcal{O}\left(m_{s}\right)$. From eq. (2.6) one can calculate the contribution from the $\mathcal{O}\left(p^{6}\right)$ LECs to $\Delta_{C T}$. It reads

$$
\left.\Delta_{C T}\right|_{C_{i}}=\frac{16}{F_{0}^{2}}\left(2 C_{12}^{r}+C_{34}^{r}\right) m_{\pi}^{2}\left(m_{K}^{2}-m_{\pi}^{2}\right) .
$$

$\mathcal{O}\left(p^{6}\right)$ calculations [27] using some estimates for the LECs $C_{12}^{r}$ and $C_{34}^{r}$ give results consistent with eq. (2.8). Strong isospin breaking as well as electromagnetic effects have also been evaluated $[27,28]$.

Another interesting quantity is the soft-kaon analog of the Callan-Treiman theorem [29]

$$
f_{0}\left(\tilde{\Delta}_{K \pi}\right)=\frac{F_{\pi}}{F_{K}}+\tilde{\Delta}_{C T}
$$

with $\tilde{\Delta}_{K \pi} \equiv-\Delta_{K \pi}$. A one loop calculation of the $\mathrm{SU}(3)$ correction $\tilde{\Delta}_{C T}$ in the isospin limit [9] gives $\tilde{\Delta}_{C T}=0.03$. This is larger than its soft-pion analog $\Delta_{C T}$, see eq. (2.8), by a factor $m_{K}^{2} / m_{\pi}^{2}$, however, rather small for a first order $\mathrm{SU}(3) \times \mathrm{SU}(3)$ breaking effect, which is expected to be of the order of about $25 \%$.

The value of $V_{u d}$, the first element of the CKM matrix is very accurately known from superallowed $0^{+} \rightarrow 0^{+}$nuclear $\beta$-decays [30]

$$
\left|V_{u d}\right|=0.97425 \pm 0.00022 .
$$

Combining this value with the experimental value of the branching ratio $\Gamma_{K_{l 2(\gamma)}} / \Gamma_{\pi_{l 2(\gamma)}}[31]$ and assuming the standard couplings of quarks to the W-boson allows to determine the ratio of the decay constants $F_{K} / F_{\pi}$. Using instead the inclusive decay rate $\Gamma_{K_{L e 3(\gamma)}}$ [31], one obtains the value of the vector form factor at zero momentum transfer $f_{+}(0)$. From these information and eqs. (2.7), (2.10), one can deduce the value of the normalized form factor at the Callan Treiman point $C \equiv f_{0}\left(\Delta_{K \pi}\right) / f_{+}(0)$ and at $\tilde{\Delta}_{K \pi}$. For the explicit formulae and more details see for example ref. [31]. One has the following updated values in the SM

$$
\begin{aligned}
\left.f_{+}(0)\right|_{S M} & =0.959 \pm 0.005 \\
F_{K} /\left.F_{\pi}\right|_{S M} & =1.192 \pm 0.006 \\
\left.\ln C\right|_{S M} & =0.2169 \pm 0.0034+\Delta_{C T} / f_{+}(0) \\
f_{0}\left(\tilde{\Delta}_{K \pi}\right) /\left.f_{+}(0)\right|_{S M} & =0.8302 \pm 0.0074+\tilde{\Delta}_{C T} / f_{+}(0) .
\end{aligned}
$$

Deviations from these SM predictions would thus be a sign of new physics. For example at NLO within the minimal not-quite decoupling electroweak low-energy effective theory (LEET) [32-34], in the light quark sector one has two combinations of parameters of spurionic origin describing the couplings of quarks to the $W$-boson to be determined from 


\begin{tabular}{|l|c|c|c|c|}
\hline & $\begin{array}{c}\text { Fit 10 [36] } \\
\text { set a }\end{array}$ & $\pi K$ Roy Steiner [37] & $\begin{array}{c}\text { Prelim. Fit All }\left(^{*}\right)[53] \\
\text { set b }\end{array}$ & Lattice [16] \\
\hline $10^{3} L_{1}^{r}$ & 0.432 & $1.05 \pm 0.12$ & $0.99 \pm 0.13$ & - \\
$10^{3} L_{2}^{r}$ & 0.735 & $1.32 \pm 0.03$ & $0.60 \pm 0.21$ & - \\
$10^{3} L_{3}^{r}$ & -2.35 & $-4.53 \pm 0.14$ & $-3.08 \pm 0.47$ & - \\
$10^{3} L_{4}^{r}$ & 0 & $0.53 \pm 0.39$ & $0.70 \pm 0.66$ & $0.33(0.13)$ \\
$10^{3} L_{5}^{r}$ & 0.97 & $3.19 \pm 2.40$ & $0.56 \pm 0.11$ & $0.93(0.073)$ \\
$10^{3} L_{6}^{r}$ & 0 & & $0.14 \pm 0.70$ & - \\
$10^{3} L_{7}^{r}$ & -0.31 & & $-0.21 \pm 0.15$ & - \\
$10^{3} L_{8}^{r}$ & 0.6 & & $0.38 \pm 0.17$ & - \\
$10^{3}\left(2 L_{6}^{r}-L_{4}^{r}\right)$ & & & & $0.032(0.062)$ \\
$10^{3}\left(2 L_{8}^{r}-L_{5}^{r}\right)$ & & & & $0.050(0.043)$ \\
\hline
\end{tabular}

Table 1. $\mathcal{O}\left(p^{4}\right)$ LECs at a scale $\mu=0.77 \mathrm{GeV}$.

experiment $[6,35]$. While the knowledge of the scalar form factor at the CT point measures one combination, its knowledge at $\tilde{\Delta}_{K \pi}$ measures the other one. A precise determination of $\Delta_{C T}$ and $\tilde{\Delta}_{C T}$ would thus help to settle the issue of the presence of right-handed couplings of quarks to the $W$-boson.

In order to have a very precise determination of $f_{+}(0)$ as well as $\Delta_{C T}$ and $\tilde{\Delta}_{C T}$, one needs to have a very precise determination of all the LECs $L_{i}^{r}$ and $C_{i}^{r}$ which enter eqs. (2.5), (2.6).

- The $L_{i}, i=1 \ldots 8$, have been determined in ref. [36] from a fit to the masses and to $K_{l 4}$-decay data from the E865 experiment, assuming that $L_{4}^{r}$ and $L_{6}^{r}$ are $1 / N_{c}$ suppressed and using as input $F_{K} / F_{\pi}=1.22$ and $m_{s} / \hat{m}=24$, with $m_{s}$ and $\hat{m}$ the strange and the average of the $u, d$ quark masses, respectively. ${ }^{1}$ Matching the dispersive results for the subthreshold expansion parameters of $\pi K$ scattering with their chiral expansion at order $p^{4}$ [37] leads to somewhat different results, especially $L_{4}^{r}$ is suggestive of a significant violation of the OZI rule in the scalar sector, see table 1. This is in agreement with a determination of some of the LECs in an analysis of $J / \psi$ decays into vector mesons and two pseudoscalars [38].

- In ref. [39] it was shown that it was possible to reproduce the values of the $L_{i}$ in terms of properties of the light meson resonances (masses and coupling constants). The idea of using resonance saturation also for the $\mathcal{O}\left(p^{6}\right)$ LECs was thus taken up and the $C_{i}$ are presently mostly estimated in that framework [40-42]. There are, however, a few problems. First the scale at which they are obtained is not known. It is usually assumed to be given by the lightest scalar nonet that survives in the large $N_{c}$ limit, $M_{S}=1.48 \mathrm{GeV}$. The value at another scale, typically the $\rho$ mass scale, is obtained using renormalization group equations. Furthermore, a test of the naturalness of the $C_{i}$ [43] shows that some of them are in fact not dominated by the resonance

\footnotetext{
${ }^{1}$ In this fit some of the $C_{i}$ are taken from resonance saturation, the others are set to zero, see ref. [15].
} 
contributions. Also the LECs we are interested in have important contributions from the scalar sector where one knows that the OZI rule is strongly violated and where the presence of the wide scalar $\sigma$ and $\kappa$ mesons makes the calculation in terms of tree level diagrams from a resonance Lagrangian not really appropriate. Considering more specifically $C_{12}^{r}$ and $C_{34}^{r}$, several calculations have been performed based on the study of the scalar form factor with $\Delta S=0[44]$ or $\Delta S=1[45,46]$. In the literature these two LECs, eq. (2.6), lie in the range $-10^{-3} \mathrm{GeV}^{-2}$ to a few $10^{-4} \mathrm{GeV}^{-2}$. The four other $\mathcal{O}\left(p^{6}\right)$ LECs $\left(C_{14}^{r}, C_{15}^{r} C_{16}^{r}\right.$ and $\left.C_{17}^{r}\right)$, eq. (2.5), needed in our study are not very well known. In ref. [47] where the $C_{i}$ have been recently determined within a quark model, one finds $C_{15}^{r}=C_{16}^{r}=0, C_{17}^{r}=0.01 \cdot 10^{-3} \mathrm{GeV}^{-2}$ and $C_{14}^{r}=-0.83 \cdot 10^{-3} \mathrm{GeV}^{-2}$ which is smaller than what is found in resonance saturation $C_{14}^{r}=-4.3 \cdot 10^{-3} \mathrm{GeV}^{-2}$.

With the progress of lattice QCD it becomes also possible to extract the LECs from a chiral extrapolation of the lattice data. Already some of the $\mathcal{O}\left(p^{4}\right)$ ones have been obtained mostly within $\mathrm{SU}(2)\left(l_{i}\right)$. Note that relations between the $\mathrm{SU}(2)$ and the $\mathrm{SU}(3)$ LECs allows to determine the $l_{i}$ from the $L_{i}[48,49]$ (for similar relations between the $C_{i}$ see ref. [50, 51]). Results from the RBC/UKQCD collaboration are shown in table 1. As can be seen from this table most of the $\mathcal{O}\left(p^{4}\right)$ LECs are still not well enough determined for a very precise test of the SM. A global fit of all the low-energy constants of Chiral Perturbation theory at next-to-next-to-leading order currently performed $[52,53]$ will hopefully help to settle the values of these LECs much more precisely. Some preliminary results [53] which differ from fit 10 by using some more recent data, by letting $L_{4}^{r}$ and $L_{6}^{r}$ free and by adding some constraints from $\pi K$ scattering show better agreement with the analysis of ref. [37] as the comparison between the second and third column of table 1 shows.

\section{Lattice}

Following the pioneering work of ref. [54] different collaborations have extracted the vector form factor at zero momentum transfer either with $N_{f}=2$ [55-58] or $N_{f}=2+1$ [13] flavours. The idea is to first evaluate the scalar form factor $f_{0}(t)$ at the momentum transfer $t_{\max }=\left(m_{K}-m_{\pi}\right)^{2}$. This can be very efficiently done calculating a double ratio of threepoint correlation functions [54]. Then a phenomenologically motivated interpolation is performed up to zero momentum transfer ${ }^{2}$ and the Ademollo-Gatto theorem is used to obtain a rather precise value for $f_{+}(0)$. Let us consider the chiral expansion of $f_{+}(0)$

$$
f_{+}(0)=1+f_{2}+f_{4}+\cdots,
$$

where $f_{n}=\mathcal{O}\left(\left(m_{K, \pi} /\left(4 \pi F_{\pi}\right)\right)^{n}\right)$ and the first term is equal to one due to gauge invariance. The Ademollo-Gatto theorem [59] states that the deviation from unity of $f_{+}(0)$ is predicted to be second order in $\mathrm{SU}(3)$ symmetry breaking, i.e. of order $\left(m_{s}-\hat{m}\right)^{2}$ so that the $\mathcal{O}\left(p^{2}\right)$

\footnotetext{
${ }^{2} \mathrm{~A}$ new technique has been developed in ref. [60] which will allow to directly simulate at $t=0$ on the lattice.
} 
term $f_{2}$ in the chiral expansion of $f_{+}(0)$ is free of any LECs. ${ }^{3}$ The different collaborations generally take this term from a one-loop ChPT calculation [9]

$$
f_{2}=-0.0227,
$$

obtained for pion, kaon and eta masses taken at their physical values and in the isospin limit and determine the difference

$$
\Delta f=f_{+}(0)-1-f_{2} .
$$

This difference contains of course all terms starting at the order $\mathcal{O}\left(p^{6}\right)$. Also used is the partially quenched expression derived in ref. [61]. An expression for $f_{2}$ using NLO SU(2) ChPT can be found in ref. [24]. The first determination of $\Delta f$ in a quark model framework gave $\Delta f=-0.016(8)[62]$.

The RBC/UKQCD collaboration for example [13, 16] simulates with $N_{f}=2+1$ flavors of dynamical domain wall quarks. In order to determine $f_{+}(0)$, they performed a simultaneous fit to both the $t$ and quark mass dependences using the ansatz

$$
f_{0}\left(t, m_{\pi}^{2}, m_{K}^{2}\right)=\frac{1+f_{2}+\left(m_{K}^{2}-m_{\pi}^{2}\right)^{2}\left(A_{0}+A_{1}\left(m_{K}^{2}+m_{\pi}^{2}\right)\right)}{1-t /\left(M_{0}+M_{1}\left(m_{K}^{2}+m_{\pi}^{2}\right)\right)^{2}} .
$$

This formula motivated by the Ademollo-Gatto theorem has four fit parameters $A_{0}, A_{1}$, $M_{0}, M_{1}$ and $f_{2}$ is the NLO term, eq. (3.3). They have also used a second order Taylor expansion as parameterization of the $t$-dependence of the form factor. ${ }^{4}$ They obtain

$$
f_{+}(0)=0.9644 \pm 0.0033_{\text {stat }} \pm 0.0037_{\text {syst }} .
$$

The same collaboration has also extracted the ratio $F_{K} / F_{\pi}[16]$

$$
F_{K} / F_{\pi}=1.205 \pm 0.018_{\text {stat }} \pm 0.062_{\text {syst }} .
$$

A summary of other lattice results can be found in refs. [31, 63-66]. In the unquenched simulations they fall in the range between 1.189 and 1.218 for the central value of $F_{K} / F_{\pi}$ and between 0.956 and 0.968 for the one of $f_{+}(0)$. While the errors on the former are very small, they are larger on the latter. All these numbers should be compared to the Standard Model values, eq. (2.12).

\section{Chiral extrapolation}

We now turn to the central point of the paper, namely the chiral extrapolation of the lattice data on $F_{K} / F_{\pi}$ and $f_{+}(0)$ based on the two-loop ChPT calculations [14]. We use

\footnotetext{
${ }^{3}$ Note, however, that despite this theorem the light quark mass difference $m_{u} \neq m_{d}$ can modify $f_{+}(0)$ to first order.

${ }^{4}$ This parameterization and the pole one are usually assumed either in lattice calculations or in most of the experimental analyses. One should note, however, that the pole parameterization has no real physical motivation in the case of the scalar form factor. Also it has been shown $[6,12]$ that in order to get a very precise parameterization of the scalar form factor in the physical region of $K_{l 3}$ decay $\left(m_{\ell}^{2}<t<t_{\max }\right)$, an expansion up to third order had to be done.
} 
the results from the RBC/UKQCD collaboration since this is the only collaboration which has calculated both these quantities with $N_{f}=2+1$ flavors. We take the data performed on the $24^{3} \times 64$ volume with an inverse lattice spacing of $a^{-1}=1.73(3) \mathrm{GeV}$ and a simulated strange quark mass, $a m_{s}=0.04$ close to its physical value. We do not correct for finite volume effects $(\mathrm{FV})$ or lattice artefacts (LA). They have been estimated for $F_{K} / F_{\pi}$ [16] where the error bars they quote for these effects are roughly equal (FV) or even larger (LA) than the statistical ones. We only included the statistical errors in our fits. Also we did not include the correlations between $F_{K}$ and $F_{\pi}$ since they are not available. Lattice results have been obtained for four values of the light quark masses which correspond to pion (first number in parenthesis) and kaon masses (second number) equal to $(0.329,0.575) \mathrm{GeV}$ (set (I)) $(0.416,0.604) \mathrm{GeV}$ (set (II)), $(0.556,0.663) \mathrm{GeV}$ (set (III)) and $(0.671,0.719) \mathrm{GeV}$. Clearly, ChPT cannot be valid at too high pion and kaon masses so we completely discard the last set in our fits and mostly use sets (I) and (II). For each pion mass they have calculated the scalar form factor at five values of $t$ going from $\sim-0.4 \mathrm{GeV}^{2}$ to $t_{\text {max }}$. Again for the fits we only use the three smallest absolute values of $t$.

A quantity $\mathcal{O}$ at two loops has typically the following form after renormalization of the pion decay constant

$$
\mathcal{O}\left(m_{\pi}, m_{K}, m_{\eta}\right)=\mathcal{O}_{L O}+\frac{\mathcal{O}_{\mathrm{NLO}}}{F_{\pi}^{2}}+\frac{\mathcal{O}_{\mathrm{NNLO}}}{F_{0}^{4}}
$$

where $\mathcal{O}_{L O}, \mathcal{O}_{\mathrm{NLO}}$ and $\mathcal{O}_{\mathrm{NNLO}}$ are the contribution at leading order (LO), next-to-leading order (NLO) and next-to-next-to-leading order (NNLO), respectively. Here, $F_{\pi}$ is the pion decay constant calculated at $\mathcal{O}\left(p^{4}\right)$ at the value of the pion mass and of the kaon mass under consideration and $F_{0}$ is the pion decay constant in the chiral SU(3) limit. When working at the physical pion and kaon masses one usually replaces everywhere the decay constant by its physical value, since the difference is of higher order. This is the procedure which has been used to determine the LECs, set (a) and (b) of table 1. If this is mostly justified for set (a) where the difference between $F_{\pi}$ and $F_{0}$ is small, this is more questionable for set (b) where $F_{0}=67.1 \mathrm{MeV}$ but allows, of course, for a better convergence of the chiral series. Also going away from the physical point the difference between $F_{\pi}$ and $F_{0}$ might become again too large for this procedure to be entirely satisfying. Here we will just replace $F_{0}$ by the physical value of $F_{\pi}$ in the NNLO term in order to be consistent with the determination of the LECs. Also to be consistent with their determination we will use eq. (2.5) for determining $F_{K} / F_{\pi}$. Again if this is justified for set (a) where the convergence of this quantity is rather good as we will see below, this is more questionable for set (b). In the expression eq. (4.1), the mass of the $\eta$ enters the NLO and NNLO terms. In the calculation of $\mathcal{O}_{\mathrm{NLO}}$ its NLO expression is used while in $\mathcal{O}_{\mathrm{NNLO}}$ the $\eta$ mass is given by the Gell-Mann-Okubo relation.

We have performed several fits to the lattice data and determined from these fits results for $f_{+}(0)$, the slope of the scalar form-factor at zero momentum transfer $\lambda_{0}, F_{K} / F_{\pi}, \Delta_{C T}$ and $\tilde{\Delta}_{C T}$. We have taken $F_{0}$ as a parameter of the fit using the value of the physical pion decay constant as input. Apart from Fit (VI) they are done with the two lattice data sets with the smallest pion values, sets (I) and (II). All the fits are done for the three smallest 


\begin{tabular}{|l|c|c|c|c|c|c|}
\hline & Fit I & Fit II & Fit III & Fit IV & Fit V & Fit VI \\
\hline$C_{12}$ & $5.77 \pm 0.56$ & $7.84 \pm 0.58$ & $4.69 \pm 0.95$ & $5.74 \pm 0.95$ & $4.69 \pm 0.56$ & $4.43 \pm 0.88$ \\
$C_{34}$ & $2.54 \pm 0.43$ & $-1.28 \pm 0.44$ & $3.76 \pm 0.95$ & $1.07 \pm 0.96$ & $3.76 \pm 0.43$ & $3.50 \pm 0.94$ \\
$C_{14}$ & $0^{*}$ & $0^{*}$ & $0.65 \pm 1.38$ & $0.71 \pm 1.42$ & $0.65^{*}$ & $-0.93 \pm 0.67$ \\
$2 C_{17}$ & $0^{*}$ & $0^{*}$ & $0.31 \pm 3.31$ & $1.92 \pm 3.36$ & $0.31^{*}$ & $4.16 \pm 1.56$ \\
$F_{0}$ & $89.8 \pm 0.1$ & $69.2 \pm 0.0$ & $89.8 \pm 0.1$ & $69.3 \pm 0.0$ & $89.8^{*}$ & $89.8 \pm 0.1$ \\
$f_{+}(0)$ & 0.956 & 0.963 & 0.956 & 0.961 & 0.956 & 0.958 \\
$F_{K} / F_{\pi}$ & 1.20 & 1.19 & 1.20 & 1.19 & 1.20 & 1.19 \\
$\ln C$ & 0.22 & 0.20 & 0.22 & 0.21 & 0.22 & 0.21 \\
$f_{0}\left(\tilde{\Delta}_{K \pi}\right)$ & 0.75 & 0.75 & 0.75 & 0.76 & 0.75 & 0.77 \\
$10^{3} \Delta_{C T}$ & 1.00 & -2.14 & 0.27 & -3.65 & 0.18 & -0.32 \\
$10^{2} \tilde{\Delta}_{C T}$ & -9.00 & -9.86 & -8.24 & -8.18 & -8.11 & -7.03 \\
$10^{3} \lambda_{0}$ & 18.08 & 17.77 & 18.24 & 17.66 & 18.18 & 16.71 \\
$\chi^{2}$ & $1.40 / 4$ & $0.96 / 4$ & $1.67 / 4$ & $1.29 / 4$ & $3.01 / 4$ & $4.8 / 7$ \\
\hline
\end{tabular}

Table 2. Result of the fits to the lattice data. The first five quantities are the parameters of the fits. The star denotes an input quantity. The $C_{i}$ 's are in units $10^{-4} \mathrm{GeV}^{-2}$ and $F_{0}$ is in $\mathrm{MeV}$. In Fits (I) and (II) the lattice data on $F_{K} / F_{\pi}$ are not included.

absolute values of $t$. The values of the LECs $L_{i}, i=1 \ldots 8$, are taken from sets (a) and (b) of table 1 and $L_{9}=5.93 \cdot 10^{-3}$ as determined from the pion charge radius. These sets correspond to a value of $m_{s} / \hat{m}=24$ as discussed previously. In ref. [52] another preliminary set is given using a somewhat larger value $m_{s} / \hat{m}=27.8$ as obtained by MILC [67] and HPQCD/UKQCD [68]. It leads to an even smaller value of $F_{0}=62.7 \mathrm{MeV}$ and will not be discussed here. The results of the fits are given in table 2. The ones from this other set are comparable to the ones of set (b).

- Fits (I) and (II) are three parameter fits of $f_{0}(t)$ using sets (a) and (b) respectively. The $C_{i}$ are the one used in the determination of the $\mathcal{O}\left(p^{4}\right)$ LECs, fit 10, namely $C_{14}^{r}=C_{15}^{r}=C_{16}^{r}=C_{17}^{r}=0$. For set (a) $F_{K} / F_{\pi}=1.22$ whereas for set (b) $F_{K} / F_{\pi}=1.19$. Slightly different values are given in the table for set (a) since, as explained below eq. (4.1) we did not use the physical value of $F_{\pi}$ in the calculation of this quantity in the NLO term but rather its NLO expression.

- Fits (III) and (IV) are combined fits of $F_{K} / F_{\pi}$ and $f_{0}(t)$ using sets (a) and (b), respectively, as in the previous fits but now the combinations $C_{14}^{r}+C_{15}^{r}$ and $C_{15}^{r}+2 C_{17}^{r}$ which appear in $F_{K} / F_{\pi}$ are left free. Since we need to determine $F_{0}$, we, in principle, need to know $C_{16}^{r}$ and the combination $C_{15}^{r}-2 C_{16}^{r}$. We will assume them equal to zero, this is consistent with the results in ref. [47]. Thus we do in fact determine $C_{14}^{r}$ and $C_{17}^{r}$.

- Fit (V): here we fix the combinations $C_{14}^{r}+C_{15}^{r}$ and $C_{15}^{r}+2 C_{17}^{r}$ from Fit (III) and we fit the quantity $\tilde{f}_{0}(t)$. 

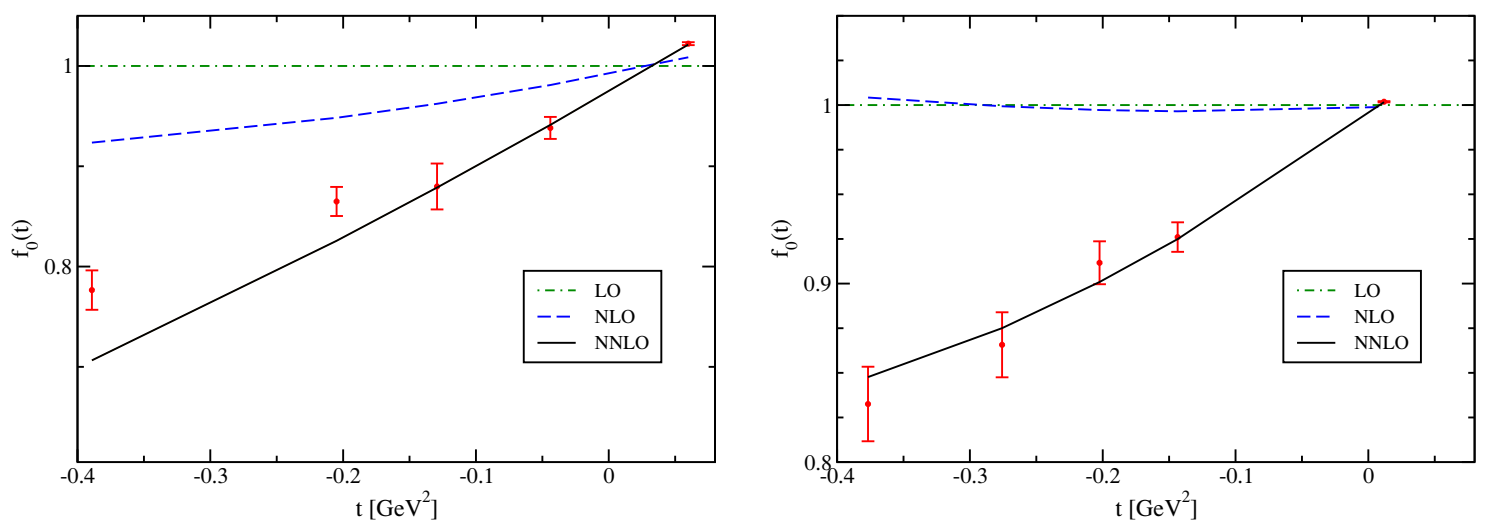

Figure 1. Momentum-dependence of the scalar form factor. The results of Fit (VI) are displayed (solid line) for set (I) (left panel) and set (III) (right panel). The convergence of the chiral expansion is also displayed: the dash-dotted line is the result at LO, the dashed line displays the one up to NLO.

- Fit (VI) is the same as Fit (III) but with the lattice data for $f_{0}(t)$ from set (III) also included.

As can be seen from table 2, we obtain very good fits of the lattice data. Fits (I) and (II), however, do not reproduce well the two lattice points for $F_{K} / F_{\pi}$ from sets (I) and (II). Fits (III) and (IV) which correspond to two very different values of $L_{4}^{r}$ are comparably good, but an order of magnitude larger value of $C_{17}^{r}$ is in fact needed in order to compensate for the larger value of $L_{4}^{r}$ in Fit (IV) compared to Fit (III). $C_{14}^{r}$ and $C_{17}^{r}$ are at least an order of magnitude smaller than what is expected from resonance saturation in the scalar sector which leads to typical values $\sim 10^{-3}$. One has for example [15]

$$
C_{14} \sim \frac{c_{d} c_{m} d_{m}}{M_{S}^{4}} \sim-4.3 \cdot 10^{-3} \mathrm{GeV}^{-2}
$$

where $c_{d}, c_{m}$ and $d_{m}$ are coefficients of the scalar chiral Lagrangian. $M_{S}$ and $d_{m}$ are obtained from the masses of the scalars $K_{0}^{*}(1430)$ and $a_{0}(980)$ and $c_{m}=0.042 \mathrm{GeV}$ and $c_{d}=0.032 \mathrm{GeV}$. The results of Fit (VI) do not differ much from Fit (III), only $C_{14}$ and $C_{17}$ are larger in absolute value and the slope of the scalar form factor is somewhat smaller. This fit is shown on figure 1 for sets (I) and (III). Even though we only fit the three smallest points in absolute value, the $t$-dependence of set (III) is remarkably well reproduced by ChPT to two loops.

Fitting $f_{0}(t)$ leads to strong anticorrelations between $C_{12}$ and $C_{34}$ on the one hand and $C_{14}+C_{15}$ and $C_{15}+2 C_{17}$ on the other one, typically of the order of -0.8 while in Fit $(\mathrm{V})$ the correlations between $C_{12}$ and $C_{34}$ are reduced by a factor of two. Also a comparison of Fits (III) and (V) shows that the error bars on these two LECs are smaller in the latter case. Thus a rather good determination of the LECs $C_{12}$ and $C_{34}$ is obtained by fitting the function $\tilde{f}_{0}$. Their order of magnitude is the one expected from resonance saturation. Note that the value obtained for $C_{12}+C_{34}$ is rather independent of the fits within one set, one gets $\sim 8 \cdot 10^{-4} \mathrm{GeV}^{-2}$ for set (a) and $\sim 6 \cdot 10^{-4} \mathrm{GeV}^{-2}$ for set (b). 
The results for $F_{K} / F_{\pi}, f_{+}(0)$ and $\ln C$ are consistent with the values obtained assuming the standard quark couplings to the $W$-boson, eq. (2.12). We refrain to give error bars here since one should have a more precise knowledge of the $L_{i}$ as well as lattice data at lower pion and kaon masses to really be able to pin down these quantities very precisely. Difference between the various sets gives an idea of the errors. The value of $\lambda_{0}$ turns out to be rather large compared to the experimental results, the lattice determination of ref. [58] or to what is obtained from the formula obtained in a dispersive parameterization of the form factor $[5,6]$

$$
\lambda_{0}=\frac{m_{\pi}^{2}}{\left(m_{K}^{2}-m_{\pi}^{2}\right)}(\ln C-G(0)), \quad G(0)=0.0398 \pm 0.0044
$$

where $G(t)$ is a dispersive integral of the phase of the form factor which is identified in the elastic region with the s-wave, $I=1 / 2 K \pi$ scattering phase according to Watson's theorem. In the analysis [5, 6] it was taken from [37] where a matching of the solution of the Roy-Steiner equations with the $K \pi \rightarrow K \pi, \pi \pi \rightarrow K \bar{K}$ and $\pi \pi \rightarrow \pi \pi$ scattering data available at higher energies has been performed. Note that in this analysis the LECs obtained, second column of table 1, are more consistent with the values used in Fit (IV), especially a large violation of the OZI rule was found. This large value of $\lambda_{0}$ can be traced back to the too large value of the combination $2 C_{12}+C_{34}$ which enters its expression within ChPT, see eq. (2.6). It is however compensated by a small curvature $\lambda_{0}^{\prime}$ leading to a value of the scalar form factor at the Callan-Treiman point in agreement with the SM value. Typically one obtains $\lambda_{0}^{\prime} \sim 1 \cdot 10^{-4}$ instead of $\sim 6 \cdot 10^{-4}$ as expected from experiments and dispersive analyses $[5,69]$. Again $C_{12}$ has a too large positive value. Note that stringent constraints on slope and curvature have recently been obtained using the method of unitarity bounds [70, 71].

Let us study the convergence of the results. In figure 1 is shown $f_{0}(t)$ as obtained in Fit (VI) at LO (dot dashed line), NLO (dashed line) and NNLO (full line). On the left-hand-side (l.h.s.) set (I) is displayed and on the right-hand-side (r.h.s.) set (III), in order to compare the dependence on the pion and the kaon masses. Clearly, as expected, the convergence of $f_{0}(t)$ worsens as one increases the absolute value of $t$ (l.h.s., set (I)) and as one increases $m_{\pi}$ and $m_{K}$. At the physical pion and kaon masses one has from Fit (III),

$$
\begin{aligned}
f_{+}(0) & =1-0.019-0.026+\ldots, \\
F_{K} / F_{\pi} & =1+0.140+0.061+\ldots, \\
f_{0}\left(\Delta_{K \pi}\right) & =1+0.139+0.063+\ldots, \\
\Delta_{C T} & =0-0.0025+0.0028+\ldots, \\
\tilde{\Delta}_{C T} & =0+0.024-0.106+\ldots,
\end{aligned}
$$


and from Fit (IV)

$$
\begin{aligned}
f_{+}(0) & =1-0.019-0.019+\ldots, \\
F_{K} / F_{\pi} & =1+0.113+0.081+\ldots, \\
f_{0}\left(\Delta_{K \pi}\right) & =1+0.110+0.081+\ldots, \\
\Delta_{C T} & =0-0.0033-0.0003+\ldots, \\
\tilde{\Delta}_{C T} & =0+0.021-0.103+\ldots,
\end{aligned}
$$

where the first, second and third terms are the $\mathcal{O}\left(p^{2}\right), \mathcal{O}\left(p^{4}\right)$ and $\mathcal{O}\left(p^{6}\right)$ contributions, respectively, and the ellipses denote terms of order $p^{8}$ and higher. Note that by definition $\Delta_{C T}$ and $\tilde{\Delta}_{C T}$ have no LO contribution. The convergence is rather good/not very good for $F_{K} / F_{\pi}$ and $f_{0}\left(\Delta_{K \pi}\right)$ for set (a) and set (b) respectively while the one for $\Delta_{C T}$ is good for set (b) and not for set (a). One should however keep in mind that the NLO correction for this last quantity is small being an $\mathrm{SU}(2) \times \mathrm{SU}(2)$ one. Also the NNLO contribution of $\Delta_{C T}$ is of the expected size of the corrections, eq. (2.8). The convergence of $f_{+}(0)$ and $\tilde{\Delta}_{C T}$ is bad whatever the set. However the convergence looks again worse than it is in reality. Indeed for both quantities the contribution at NLO is smaller than naively expected. For $f_{+}(0)$ this is essentially due to the Ademollo-Gatto theorem, as we have seen in the previous section. In both cases the NNLO term is of the expected size. Let us look in a bit more details at the diverse contributions for $f_{+}(0), F_{K} / F_{\pi}$ and $f_{0}\left(\Delta_{K \pi}\right)$. One has for Fit (III)

$$
\begin{aligned}
f_{+}(0) & =1+(-0.019+0.000)+(0.012-0.003-0.034)+\ldots, \\
F_{K} / F_{\pi} & =1+(0.057+0.083)+(-0.005+0.045+0.021)+\ldots, \\
f_{0}\left(\Delta_{K \pi}\right) & =1+(0.055+0.083)+(-0.001+0.047+0.017)+\ldots,
\end{aligned}
$$

and for Fit (IV)

$$
\begin{aligned}
f_{+}(0) & =1+(-0.027+0.008)+(0.012-0.002-0.029)+\ldots, \\
F_{K} / F_{\pi} & =1+(0.086+0.027)+(-0.005+0.078+0.009)+\ldots, \\
f_{0}\left(\Delta_{K \pi}\right) & =1+(0.083+0.026)+(-0.001+0.063+0.019)+\ldots
\end{aligned}
$$

The first brackets give the contribution from the loops and the $L_{i}$ at fourth order and the second brackets represent the one at sixth order from the two-loops, the one-loop with one $L_{i}$ insertion plus tree graphs with two $L_{i}$ and the tree graphs $\sim C_{i}$, in order. One sees that the large contribution of $f_{+}(0)$ at NNLO is due to big corrections of the dimension six operators, as was the case for the slope and the curvature, see the discussion before. It could be that the corresponding LECs $C_{i}$ are larger than they are in nature mocking up some higher order effects. The contributions from the two-loop and the one-loop $\sim L_{i}$ topologies do converge. In the case of $F_{K} / F_{\pi}$ and $f_{0}\left(\Delta_{K \pi}\right)$ it is the terms proportional to $L_{i}$ which are responsible for their not so good convergence in the case of set (b), explaining the difference between the two sets. Let us consider also the convergence of $F_{K} / F_{\pi}$ at larger pion and kaon masses. One has for Fit (IV)

$$
\begin{aligned}
F_{K} / F_{\pi} & =1+0.043+0.093+\ldots=1.136+\ldots, & & \text { set }(\mathrm{I}) \\
& =1+0.023+0.076+\ldots=1.099+\ldots, & & \text { set }(\mathrm{II}) .
\end{aligned}
$$


For comparison the lattice data are:

$$
\begin{array}{rlr}
F_{K} / F_{\pi} & =1.134 \pm 0.011, \quad & \operatorname{set}(\mathrm{I}) \\
& =1.101 \pm 0.010, \quad & \operatorname{set}(\mathrm{II}) .
\end{array}
$$

As already stated for the scalar form factor and as expected, the convergence gets worse when increasing the values of $m_{\pi}$ and $m_{K}$. This bad convergence could be an artefact of the use of lattice data obtained at still too high pion and kaon masses for ChPT to really be valid.

\section{Conclusion}

We have done here a first exploratory study using a two-loop ChPT calculation to fit the lattice data. Certainly finite volume effects for example should be taken into account in a more refined treatment. However, before this can be done, a better knowledge of the $L_{i}$ are necessary and more lattice data at smaller masses are needed. This is important for checking the convergence of the $\mathrm{SU}(3)$ ChPT calculations as well as for a more precise determination of the quantities studied here. Also if the result of set (a) is not very sensitive to the treatment of the NNLO term, see discussion below eq. (4.1), this is clearly not the case for set (b) and our results here are certainly not the final ones. Indeed, if large values for $L_{4}^{r}$ and $L_{6}^{r}$ as expected from a large violation of the OZI rule were confirmed in the future then the use of standard ChPT as done here would not really be appropriate. A way of solving the problem could be for example to work within resummed ChPT $[72,73]$. A study along this line is in progress [74].

\section{Acknowledgments}

We are extremely grateful to Johan Bijnens for giving us his programs for calculating the scalar and vector form factors. Without his help this work would not have been possible. We would like to thank the RBC/UKQCD collaboration for providing us with the lattice data on the scalar form factor. We are grateful to Sebastien Descotes-Genon, Christoph Haefeli, Ilaria Jemos, Andreas Jüttner and Ulf-G. Meißner for interesting discussions and useful comments. This work has been supported in part by the EU contract MRTN-CT2006-035482 ("Flavianet"), the European Community-Research Infrastructure Integrating Activity "Study of Strongly Interaction Matter" (acronym Hadron-Physics2, Grant Agreement n. 227431) under the Seventh Framework Programme of the EU, by MEC (Spain) under grant FPA2007-60323 and by the Spanish Consolider-Ingenio 2010 Programme CPAN (CSD2007-00042).

Open Access. This article is distributed under the terms of the Creative Commons Attribution Noncommercial License which permits any noncommercial use, distribution, and reproduction in any medium, provided the original author(s) and source are credited. 


\section{References}

[1] S.R. Sharpe, Applications of chiral perturbation theory to lattice QCD, hep-lat/0607016 [SPIRES].

[2] U.-G. Meissner and G. Schierholz, Lattice QCD, chiral perturbation theory and hadron phenomenology, hep-ph/0611072 [SPIRES].

[3] V. Bernard, Chiral Perturbation Theory and Baryon Properties, Prog. Part. Nucl. Phys. 60 (2008) 82 [arXiv:0706.0312] [SPIRES].

[4] Flavianet Working Group on Kaon Decays, M. Antonelli et al., Precision tests of the Standard Model with leptonic and semileptonic kaon decays, arXiv:0801.1817 [SPIRES] (http://www.lnf.infn.it/wg/vus/).

[5] V. Bernard, M. Oertel, E. Passemar and J. Stern, $K_{L \mu 3}$ decay: A stringent test of right-handed quark currents, Phys. Lett. B 638 (2006) 480 [hep-ph/0603202] [SPIRES].

[6] V. Bernard, M. Oertel, E. Passemar and J. Stern, Dispersive representation and shape of the $K_{l 3}$ form factors: robustness, Phys. Rev. D 80 (2009) 034034 [arXiv:0903.1654] [SPIRES].

[7] V. Bernard, M. Oertel, E. Passemar and J. Stern, Tests of non-standard electroweak couplings of right-handed quarks, JHEP 01 (2008) 015 [arXiv:0707.4194] [SPIRES].

[8] O. Deschamps et al., The Two Higgs Doublet of Type II facing flavour physics data, arXiv:0907.5135 [SPIRES].

[9] J. Gasser and H. Leutwyler, Low-Energy Expansion of Meson Form-Factors, Nucl. Phys. B 250 (1985) 517 [SPIRES].

[10] NA48 collaboration, A. Lai et al., Measurement of $\mathrm{K}_{\mu 3}^{0}$ form factors, Phys. Lett. B 647 (2007) 341 [hep-ex/0703002] [SPIRES].

[11] KLOE collaboration, F. Ambrosino et al., Measurement of the $K_{L} \rightarrow \pi \mu \nu$ form factor parameters with the KLOE detector, JHEP 12 (2007) 105 [arXiv:0710.4470] [SPIRES].

[12] KTEV collaboration, E. Abouzaid et al., Dispersive analysis of $K_{L \mu 3}$ and $K_{L e 3}$ scalar and vector form factors using KTeV data, arXiv:0912.1291 [SPIRES].

[13] P.A. Boyle et al., $K_{l 3}$ semileptonic form factor from $2+1$ flavour lattice $Q C D$, Phys. Rev. Lett. 100 (2008) 141601 [arXiv:0710.5136] [SPIRES].

[14] J. Bijnens and P. Talavera, $K_{l 3}$ decays in chiral perturbation theory, Nucl. Phys. B 669 (2003) 341 [hep-ph/0303103] [SPIRES].

[15] G. Amoros, J. Bijnens and P. Talavera, Two-point functions at two loops in three flavour chiral perturbation theory, Nucl. Phys. B 568 (2000) 319 [hep-ph/9907264] [SPIRES].

[16] RBC-UKQCD collaboration, C. Allton et al., Physical Results from $2+1$ Flavor Domain Wall QCD and SU(2) Chiral Perturbation Theory, Phys. Rev. D 78 (2008) 114509 [arXiv:0804.0473] [SPIRES].

[17] B. Moussallam, $N(f)$ dependence of the quark condensate from a chiral sum rule, Eur. Phys. J. C 14 (2000) 111 [hep-ph/9909292] [SPIRES].

[18] B. Moussallam, Flavor stability of the chiral vacuum and scalar meson dynamics, JHEP 08 (2000) 005 [hep-ph/0005245] [SPIRES].

[19] S. Descotes-Genon, L. Girlanda and J. Stern, Paramagnetic effect of light quark loops on chiral symmetry breaking, JHEP 01 (2000) 041 [hep-ph/9910537] [SPIRES].

[20] S. Descotes-Genon and J. Stern, Vacuum fluctuations of anti- $q$ q and values of low-energy constants, Phys. Lett. B 488 (2000) 274 [hep-ph/0007082] [SPIRES]. 
[21] S. Descotes-Genon, How far can you go? Surprises and pitfalls in three-flavour chiral extrapolations, PoS(LATTICE 2007) 070 [arXiv:0709.0265] [SPIRES].

[22] A. Roessl, Pion kaon scattering near the threshold in chiral SU(2) perturbation theory, Nucl. Phys. B 555 (1999) 507 [hep-ph/9904230] [SPIRES].

[23] M. Frink, B. Kubis and U.-G. Meissner, Analysis of the pion kaon sigma-term and related topics, Eur. Phys. J. C 25 (2002) 259 [hep-ph/0203193] [SPIRES].

[24] RBC collaboration and UKQCD collaboration, J.M. Flynn and C.T. Sachrajda, SU(2) chiral prturbation theory for $K_{l 3}$ decay amplitudes, Nucl. Phys. B $\mathbf{8 1 2}$ (2009) 64 [arXiv:0809.1229] [SPIRES].

[25] C.G. Callan and S.B. Treiman, Equal Time Commutators and K Meson Decays, Phys. Rev. Lett. 16 (1966) 153 [SPIRES].

[26] R.F. Dashen and M. Weinstein, Theorem on the form-factors in k-l-3 decay, Phys. Rev. Lett. 22 (1969) 1337 [SPIRES].

[27] J. Bijnens and K. Ghorbani, Isospin breaking in $K \pi$ vector form-factors for the weak and rare decays $K_{\ell 3}, K \rightarrow \pi \nu \bar{\nu}$ and $K \rightarrow \pi \ell^{+} \ell^{-}$, arXiv:0711.0148 [SPIRES].

[28] A. Kastner and H. Neufeld, The $K_{l 3}$ scalar form factors in the standard model, Eur. Phys. J. C 57 (2008) 541 [arXiv:0805.2222] [SPIRES].

[29] R. Oehme, Current Algebras and the Suppression of Leptonic Meson Decays with $\Delta S=1$, Phys. Rev. Lett. 16 (1966) 215.

[30] J.C. Hardy and I.S. Towner, Superallowed $0^{+} \rightarrow 0^{+}$nuclear beta decays: A new survey with precision tests of the conserved vector current hypothesis and the standard model, Phys. Rev. C 79 (2009) 055502 [arXiv: 0812.1202] [SPIRES].

[31] M. Antonelli et al., Flavor Physics in the Quark Sector, arXiv:0907.5386 [SPIRES].

[32] J. Hirn and J. Stern, The role of spurions in Higgs-less electroweak effective theories, Eur. Phys. J. C 34 (2004) 447 [hep-ph/0401032] [SPIRES].

[33] J. Hirn and J. Stern, Anomaly-matching and Higgs-less effective theories, JHEP 09 (2004) 058 [hep-ph/0403017] [SPIRES].

[34] J. Hirn and J. Stern, Lepton-number violation and right-handed neutrinos in Higgs-less effective theories, Phys. Rev. D 73 (2006) 056001 [hep-ph/0504277] [SPIRES].

[35] J. Stern, Chiral dynamics beyond the standard model, Nucl. Phys. Proc. Suppl. 174 (2007) 109 [hep-ph/0611127] [SPIRES].

[36] G. Amoros, J. Bijnens and P. Talavera, QCD isospin breaking in meson masses, decay constants and quark mass ratios, Nucl. Phys. B 602 (2001) 87 [hep-ph/0101127] [SPIRES].

[37] P. Buettiker, S. Descotes-Genon and B. Moussallam, A re-analysis of $\pi K$ scattering a la Roy and Steiner, Eur. Phys. J. C 33 (2004) 409 [hep-ph/0310283] [SPIRES].

[38] T.A. Lahde and U.-G. Meissner, Improved Analysis of $J / \psi$ Decays into a Vector Meson and Two Pseudoscalars, Phys. Rev. D 74 (2006) 034021 [hep-ph/0606133] [SPIRES].

[39] G. Ecker, J. Gasser, A. Pich and E. de Rafael, The Role of Resonances in Chiral Perturbation Theory, Nucl. Phys. B 321 (1989) 311 [SPIRES].

[40] V. Cirigliano et al., Towards a consistent estimate of the chiral low-energy constants, Nucl. Phys. B 753 (2006) 139 [hep-ph/0603205] [SPIRES].

[41] V. Cirigliano et al., The $\langle S P P>$ Green function and $\mathrm{SU}$ (3) breaking in $K(13)$ decays, JHEP 04 (2005) 006 [hep-ph/0503108] [SPIRES]. 
[42] G. Ecker, Chiral low-energy constants, Acta Phys. Polon. B 38 (2007) 2753 [hep-ph/0702263] [SPIRES].

[43] K. Kampf and B. Moussallam, Tests of the naturalness of the coupling constants in ChPT at order $p^{6}$, Eur. Phys. J. C 47 (2006) 723 [hep-ph/0604125] [SPIRES].

[44] J. Bijnens and P. Dhonte, Scalar form factors in SU(3) chiral perturbation theory, JHEP 10 (2003) 061 [hep-ph/0307044] [SPIRES].

[45] M. Jamin, J.A. Oller and A. Pich, Order $p^{6}$ chiral couplings from the scalar $K \pi$ form-factor, JHEP 02 (2004) 047 [hep-ph/0401080] [SPIRES].

[46] V. Bernard and E. Passemar, Matching Chiral Perturbation Theory and the Dispersive

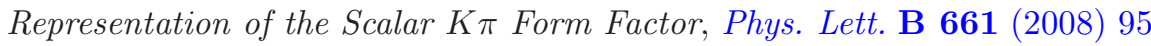
[arXiv:0711.3450] [SPIRES].

[47] S.-Z. Jiang, Y. Zhang, C. Li and Q. Wang, Computation of the $p^{6}$ order chiral Lagrangian coefficients from the underlying theory of QCD, arXiv:0907.5229 [SPIRES].

[48] J. Gasser and H. Leutwyler, Chiral Perturbation Theory: Expansions in the Mass of the Strange Quark, Nucl. Phys. B 250 (1985) 465 [SPIRES].

[49] J. Gasser, C. Haefeli, M.A. Ivanov and M. Schmid, Integrating out strange quarks in ChPT, Phys. Lett. B 652 (2007) 21 [arXiv:0706.0955] [SPIRES].

[50] J. Gasser, C. Haefeli, M.A. Ivanov and M. Schmid, Integrating out strange quarks in ChPT: terms at order $p^{6}$, Phys. Lett. B 675 (2009) 49 [arXiv:0903.0801] [SPIRES].

[51] J. Gasser, C. Haefeli, M.A. Ivanov and M. Schmid, Relations between SU(2)- and $\mathrm{SU}(3)-L E C s$ in chiral perturbation theory, arXiv:0910.4880 [SPIRES].

[52] J. Bijnens and I. Jemos, Determination of Low Energy Constants and testing Chiral Perturbation Theory at order $p^{6}$ (NNLO), arXiv:0909.4477 [SPIRES].

[53] J. Bijnens and I. Jemos, Fitting Low Energy Constants at Next to Next to Leading order in Chiral Perturbation Theory, talk given at Euroflavour'09, Annual Workshop of the European Flavour Physics Network FLAVIAnet, INFN, Bari Italy (2009).

[54] D. Becirevic et al., The $K \rightarrow \pi$ vector form factor at zero momentum transfer on the lattice, Nucl. Phys. B 705 (2005) 339 [hep-ph/0403217] [SPIRES].

[55] JLQCD collaboration, N. Tsutsui et al., Kaon semileptonic decay form factors in two-flavor QCD, PoS (LAT2005) 357 [hep-lat/0510068] [SPIRES].

[56] C. Dawson, T. Izubuchi, T. Kaneko, S. Sasaki and A. Soni, Vector form factor in $K_{l 3}$ semileptonic decay with two flavors of dynamical domain-wall quarks, Phys. Rev. D 74 (2006) 114502 [hep-ph/0607162] [SPIRES].

[57] The QCDSF collaboration, D. Brommel et al., Kaon semileptonic decay form factors from $N_{f}=2$ non-perturbatively $O(a)$-improved Wilson fermions, PoS (LATTICE 2007) 364 [arXiv:0710.2100] [SPIRES].

[58] V. Lubicz, F. Mescia, S. Simula, C. Tarantino and F.T.E. collaboration, $K \rightarrow$ pion Semileptonic Form Factors from Two-Flavor Lattice QCD, Phys. Rev. D 80 (2009) 111502 [arXiv: 0906.4728] [SPIRES].

[59] M. Ademollo and R. Gatto, Nonrenormalization Theorem for the Strangeness Violating Vector Currents, Phys. Rev. Lett. 13 (1964) 264 [SPIRES].

[60] P.A. Boyle, J.M. Flynn, A. Juttner, C.T. Sachrajda and J.M. Zanotti, Hadronic form factors in lattice QCD at small and vanishing momentum transfer, JHEP 05 (2007) 016 [hep-lat/0703005] [SPIRES]. 
[61] D. Becirevic, G. Martinelli and G. Villadoro, The Ademollo-Gatto theorem for lattice semileptonic decays, Phys. Lett. B 633 (2006) 84 [hep-lat/0508013] [SPIRES].

[62] H. Leutwyler and M. Roos, Determination of the Elements V(us) and V(ud) of the Kobayashi-Maskawa Matrix, Z. Phys. C 25 (1984) 91 [SPIRES].

[63] The FLAG working group, G. Colangelo, Making lattice results accessible to phenomenologists, talk given at the Kaon'09 conference PoS (KAON09) 029 (2009).

[64] The FLAG working group, G. Colangelo, Status report of the FLAG activities, talk given at Euroflavour'09, Annual Workshop of the European Flavour Physics Network FLAVIAnet, INFN, Bari Italy (2009).

[65] H. Leutwyler, On the interface between lattice results and Chiral Perturbation Theory, talk given at the Colloquium in memory of J. Stern, Paris France.

[66] The FLAG working group, in preparation.

[67] The MILC collaboration, A. Bazavov et al., Results from the MILC collaboration's SU(3) chiral perturbation theory analysis, PoS (LAT2009) 079 [arXiv:0910.3618] [SPIRES].

[68] HPQCD collaboration, C. Aubin et al., First determination of the strange and light quark masses from full lattice QCD, Phys. Rev. D 70 (2004) 031504 [hep-lat/0405022] [SPIRES].

[69] M. Jamin, J.A. Oller and A. Pich, Scalar $K \pi$ form factor and light quark masses, Phys. Rev. D 74 (2006) 074009 [hep-ph/0605095] [SPIRES].

[70] G. Abbas and B. Ananthanarayan, Improved bounds on the radius and curvature of the $K \pi$ scalar form factor and implications to low-energy theorems, Eur. Phys. J. A 41 (2009) 7 [arXiv: 0905.0951] [SPIRES].

[71] G. Abbas, B. Ananthanarayan, I. Caprini, I.S. Imsong and S. Ramanan, Stringent constraints on the scalar $K \pi$ form factor from analyticity, unitarity and low-energy theorems, arXiv: 0912.2831 [SPIRES].

[72] S. Descotes-Genon, N.H. Fuchs, L. Girlanda and J. Stern, Resumming QCD vacuum fluctuations in three-flavour chiral perturbation theory, Eur. Phys. J. C 34 (2004) 201 [hep-ph/0311120] [SPIRES].

[73] S. Descotes-Genon, Low-energy $\pi \pi$ and $\pi K$ scatterings revisited in three-flavour resummed chiral perturbation theory, Eur. Phys. J. C 52 (2007) 141 [hep-ph/0703154] [SPIRES].

[74] V. Bernard, S. Descotes-Genon and G. Toucas, in preparation. 